\title{
Correction to: Refractory anti-NMDAR encephalitis successfully treated with bortezomib and associated movements disorders controlled with tramadol: a case report with literature review
}

\author{
Serena Marita Lazzarin ${ }^{1,2} \cdot$ Marco Vabanesi $^{1,2}$ (1) $\cdot$ Giordano Cecchetti $^{1,2}$ (1) $\cdot$ Raffaella Fazio $^{1} \cdot$ \\ Giovanna Franca Fanelli ${ }^{2}$. Maria Antonietta Volonté ${ }^{1}$ - Angela Genchi ${ }^{1}$. Antonino Giordano ${ }^{1}$ (1) . \\ Vittorio Martinelli $i^{1}$ (1) Sergio Colombo ${ }^{3} \cdot$ Paolo Beccaria $^{3} \cdot$ Milena Mucci $^{3}$. Jacopo Peccatori ${ }^{4}$. \\ Massimo Filippi ${ }^{1,2,5,6}$ (D) $\cdot$ Fabio Minicucci ${ }^{2}$
}

Published online: 8 January 2021

(c) Springer-Verlag GmbH Germany, part of Springer Nature 2021

\section{Correction to: Journal of Neurology (2020) 267:2462-2468 https://doi.org/10.1007/s00415-020-09988-w}

The authors would like to correct some errors in the publication of the original article. The corrected and updated details are given below for your reading.

In "Case Report" section, the first sentence of the last paragraph should be:

"After three weeks from the last immunosuppressive treatment, a rescue therapy with bortezomib (four doses, $1.3 \mathrm{mg} / \mathrm{m}^{2}$ each, on days $\left.1-4-8-11\right)$ and dexamethasone was administered, followed by other two cycles; the third cycle was interrupted after the first dose due to respiratory infection from $P$. aeruginosa."

In "Case Report" section, the penultimate sentence of the last paragraph should be:

The original article can be found online at https://doi.org/10.1007/ s00415-020-09988-w.

Massimo Filippi

filippi.massimo@hsr.it

1 Neurology Unit, IRCCS San Raffaele Scientific Institute, Milan, Italy

2 Neurophysiology Unit, IRCCS San Raffaele Scientific Institute, Milan, Italy

3 General Intensive Care Unit, IRCCS San Raffaele Scientific Institute, Milan, Italy

4 Hematology and Bone Marrow Transplantation Unit, IRCCS San Raffaele Scientific Institute, Milan, Italy

5 Neuroimaging Research Unit, Institute of Experimental Neurology, Division of Neuroscience, IRCCS San Raffaele Scientific Institute, Via Olgettina, 60, 20132 Milan, Italy

6 Vita-Salute San Raffaele University, Milan, Italy
"After a cycle of combined therapy with bortezomib (4 weekly doses, $1.3 \mathrm{mg} / \mathrm{m}^{2}$ each), daratumumab (4 weekly doses, $16 \mathrm{mg} / \mathrm{kg}$ each) and dexamethasone (20 mg twice weekly, on days 1-2, for 4 weeks), at 6 months from onset, she was admitted to the rehabilitation clinic. She was able to walk with assistance, interacted spontaneously with other people and showed moderate dysarthria. Ten months after the symptom onset, a Memory Clinic visit reported persistent neurocognitive dysfunctions: she was unable to use cash or public transportation alone, and she needed help in taking care of the house."

In "Discussion-Bortezomib in refractory anti-NMDAR encephalitis" section, the first two sentences of the last paragraph should be:

"In our case, three cycles of bortezomib/dexamethasone were prescribed according to the protocol approved for multiple myeloma [35], followed by one cycle of bortezomib/ daratumumab/dexamethasone; the drugs were well tolerated, except for increased risk of infections. The patient showed a slight, progressive amelioration in consciousness after the second bortezomib cycle and further clinical improvement after combination therapy with daratumumab. At 6-month follow-up from symptoms onset, she reached mRS level of 4."

In "Conclusion" section, the second sentence of the third paragraph should be:

"Bortezomib, a specific proteasome inhibitor targeting plasma cells, possibly combined with daratumumab, a CD38 inhibitor with further effects on $T_{\text {reg }}$ and NK cells, may prove useful as a rescue therapy in severe anti-NMDAR encephalitis cases."

Figure 3 should be replaced by the following one: 


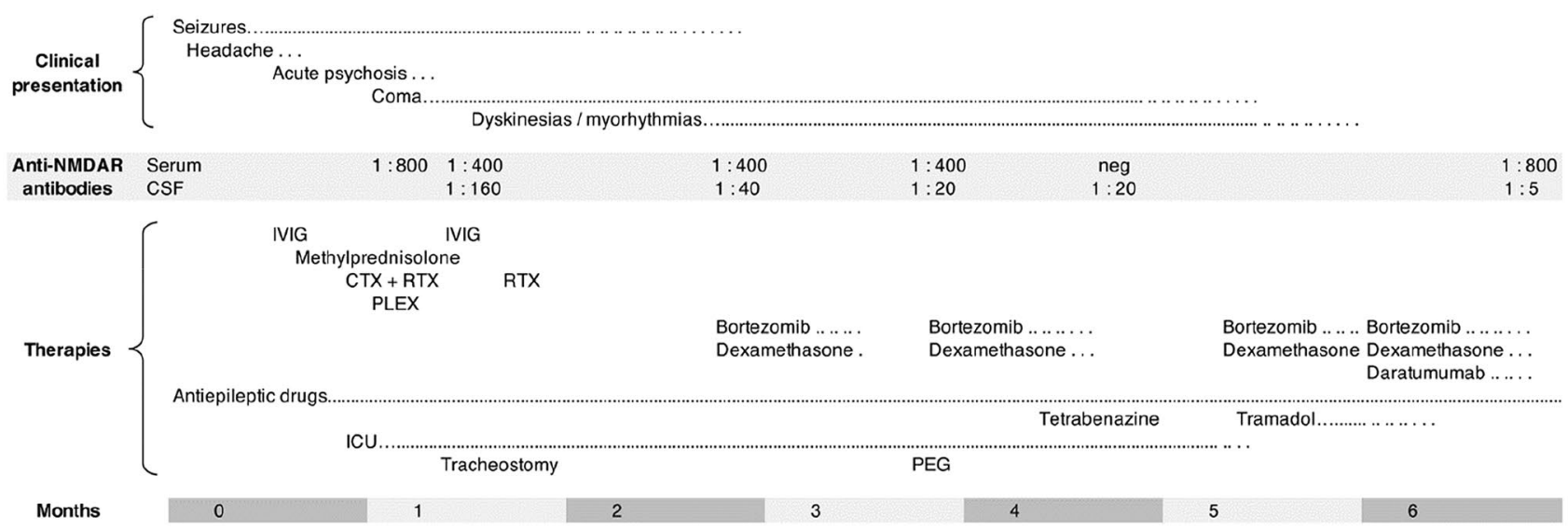

Fig. 3 Timeline of main clinical data, treatments and antibody titres. Legend. CSF cerebrospinal fluid, IVIG intravenous immunoglobulins, CTX cyclophosphamide, $R T X$ rituximab, $I C U$ intensive care unit, $P E G$ percutaneous endoscopic gastrostomy 